\title{
Fast magnetic reconnection in the plasmoid-dominated regime
}

\author{
D. A. Uzdensky, ${ }^{1,2}$ N. F. Loureiro, ${ }^{3,2}$ and A. A. Schekochihin ${ }^{4,2}$ \\ ${ }^{1}$ Center for Integrated Plasma Studies, University of Colorado, Boulder CO 80309, USA \\ ${ }^{2}$ Isaac Newton Institute for Mathematical Sciences, Cambridge, CB3 OEH, UK \\ ${ }^{3}$ Associação EURATOM/IST, Instituto de Plasmas e Fusão Nuclear - Laboratório Associado, \\ Instituto Superior Técnico, 1049-001 Lisboa, Portugal \\ ${ }^{4}$ Rudolf Peierls Centre for Theoretical Physics, University of Oxford, Oxford OX1 3NP, UK
}

(Dated: November 6, 2018)

\begin{abstract}
A conceptual model of resistive magnetic reconnection via a stochastic plasmoid chain is proposed. The global reconnection rate is shown to be independent of the Lundquist number. The distribution of fluxes in the plasmoids is shown to be an inverse square law. It is argued that there is a finite probability of emergence of abnormally large plasmoids, which can disrupt the chain (and may be responsible for observable large abrupt events in solar flares and sawtooth crashes). A criterion for the transition from magnetohydrodynamic to collisionless regime is provided.
\end{abstract}

PACS numbers: 52.35.Vd, 94.30.cp, 96.60.Iv, 52.35.Py

Introduction. Magnetic reconnection is the process of topological rearrangement of magnetic field, resulting in a conversion of magnetic energy into various forms of plasma energy [1]. It is believed to cause solar flares and has been studied in tokamaks [2], dedicated laboratory experiments 3] and measured in situ in the Earth's magnetosphere [4]. The basic conceptual underpinnings of the modern understanding of resistive reconnection can be summarised in three points: (i) generic X-point configurations are unstable and collapse into current layers [5, 6]; (ii) the structure of resistive current layers is well described by the Sweet-Parker (SP) model [7]: if $B_{0}$ is the upstream magnetic field, $V_{A}=B_{0} / \sqrt{4 \pi \rho}$ is the Alfvén speed ( $\rho$ the plasma density), $L$ the length of the layer, $\eta$ the magnetic diffusivity, and $S \equiv V_{A} L / \eta$ the Lundquist number, then the layer thickness is $\delta \sim L / \sqrt{S}$, the outflow velocity is $V_{A}$, and the reconnection rate is $c E \sim V_{A} B_{0} / \sqrt{S}$ — "slow" because it depends on $S$, which is very large in most natural systems; (iii) when $S$ exceeds a critical value $S_{c} \sim 10^{4}$, the SP layers are linearly unstable [8] and break up into secondary islands, or plasmoids [9]. This fact has emerged as a defining feature of numerical simulations of reconnection as they have broken through the $S_{c}$ barrier [6, 9 16]. It seems that high- $S$ reconnection generically occurs via a chain of plasmoids, born, growing, coalescing, and being ejected in a stochastic fashion [17, 18]. Importantly, recent numerical evidence [11, 13 16] suggests that plasmoid reconnection is "fast", i.e., independent of $S$.

In this Letter, we propose a conceptual model of a resistively reconnecting incompressible plasmoid chain and infer the following basic properties: (i) the global reconnection rate is independent of $S$; (ii) the chain is described by a power law (inverse square) distribution of plasmoid fluxes and sizes; (iii) there is a finite probability of abnormally large plasmoids that can disrupt the chain. We also provide a criterion for a transition from the fast resistive MHD regime to a faster collisionless regime.
Plasmoid chain: general physical picture and key assumptions. We envision the plasmoid-dominated reconnection layer in a statistical steady state as a chain of plasmoids of various sizes separated by small current sheets. There is an underlying velocity gradient along the chain: all plasmoids are moving outwards as the reconnecting flux is coming in. The plasmoids differ greatly in size and hence should not be regarded as equal members of the chain - the chain has a hierarchical structure. Consider the global current layer of half-length $L$. We may call the largest plasmoids in it the secondary ones. Any pair of two adjacent secondary plasmoids transforms the region between them into a secondary reconnection layer of length $2 L^{(2)} \ll 2 L$ with end-toend longitudinal velocity difference of $\sim 2 V_{A}$. Then, the characteristic ejection time for tertiary plasmoids created by the plasmoid instability [8] in this secondary layer is $\sim \tau_{A}^{(2)}=L^{(2)} / V_{A}$, much shorter than the global Alfvén time $\tau_{A}=L / V_{A}$. Thus, the tertiary plasmoids are ejected from the secondary layer relatively rapidly and their typical size remains smaller than that of the secondary plasmoids. Upon ejection, the tertiary plasmoids coalesce with the larger secondary ones, contributing to their growth. Thus, the secondary layer itself becomes a plasmoid-dominated reconnection layer. A similar argument can be applied to the tertiary layers and so on. We thus get a self-similar hierarchy of plasmoids and interplasmoid current layers that are themselves complex chains of next-generation plasmoids [14, 16, 17].

The smallest elementary structure in the chain is the "critical layer" — an SP layer marginally stable to the plasmoid instability $\left(S=S_{c} \sim 10^{4}\right)$. Its key parameters - the length $L_{c}=S_{c} \eta / V_{A}$, the thickness $\delta_{c}=L_{c} / \sqrt{S_{c}}$, and the reconnection rate $c E_{c}=B_{0} V_{A} / \sqrt{S_{c}}$ - depend only on $\eta$ and $V_{A}$ but not on the system size $L$ [16]. We expect that the smallest current layers found in the system are never much shorter or much longer than the critical length $L_{c}$ and their reconnection rate always hov- 
ers around $E_{c}$. Indeed, because of the underlying velocity gradient, the secondary current layer between any two adjacent plasmoids is continuously stretched. When its length exceeds $L_{c}$, this layer becomes slightly supercritical to the plasmoid instability [8] and produces a new plasmoid flanked by two new X-points. This new plasmoid grows rapidly and reaches the critical size at which the two X-points on its sides undergo an X-point collapse [5, 6], turning promptly into two new current sheets. This process repeats continuously. We assume that this effective splitting of a super-critical inter-plasmoid layer into two occurs faster than the typical stretching time of that layer; then the lengths of the resulting new layers are always somewhat smaller than $L_{c}$. Thus, the plasmoid chain contains $N \sim L / L_{c} \sim S / S_{c}$ plasmoids (of all generations) separated by nearly critical current layers 23]. The hierarchy of plasmoid-dominated layers is truncated at the critical layer.

We can now formalize the above physical picture in terms of the following assumptions (or conjectures), expected to hold on average:

(I) The X-point collapses and layer-splitting instabilities are sufficiently fast (Alfvénic or super-Alfvénic), so on average any two immediately adjacent plasmoids are separated by a critical layer.

(II) The upstream reconnecting field in each interplasmoid layer is equal to the global reconnecting field $B_{0}$. This implies that the outflows into all plasmoids are Alfvénic, with velocity $V_{A}$. This is easy to show via a theoretical analysis of the acceleration of plasmoids along the layer by the magnetic tension of the associated open flux [19], but here will be assumed without proof. It then follows that the mean flow in the global layer (and in each sub-layer) is roughly Hubble-like, $v_{y} \sim V_{A} y / L$ ( $x$ and $y$ are the directions across and along the layer).

(III) Plasmoids do not saturate before they are ejected from the current layers in which they are embedded into larger plasmoids flanking these layers.

Assumptions I-II appear to be supported by numerical evidence but need to be checked systematically. Assumption III will be verified a posteriori in our theory.

Effective reconnection rate. A key question in any reconnection study is that of the reconnection rate. Because of the inherently non-steady nature of plasmoid reconnection, we are interested in the effective timeaveraged (global) rate of transfer of magnetic flux from the upstream to the downstream region. To find it, consider a simple chain of secondary plasmoids and current sheets. The fact that the secondary current layers in the hierarchical picture are not simple SP sheets but, instead, complex plasmoid-dominated layers is not important here; we will just view them as effective reconnection regions with reconnection rate $E^{(2)}$. Our goal is to relate the effective global reconnection rate $E_{\text {eff }}$ to $E^{(2)}$.

Note that a fully closed magnetic island carries no net reconnected flux $\left(B_{x}\right)$. The only reconnected flux actu-

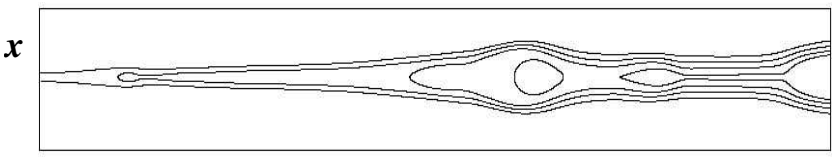

$\boldsymbol{y}$

FIG. 1: Contour plot of $\Psi$ from an MHD simulation at $S=$ $3 \cdot 10^{5}$ (detail), illustrating the open magnetic flux.

ally carried out is the open flux threading the midplane $x=0$ between the secondary current sheets and plasmoids and ejected from the layer with them. This flux accumulates because the plasmoid chain is not quite uniform in $y$ : the reconnection rates at the two $\mathrm{X}$-points on both sides of any given plasmoid slightly differ (because $B_{0}$ decreases outward along $y$ ), resulting in the growth of open flux on the faster-reconnecting (smaller- $|y|$ ) side of the plasmoid. Thus, strictly speaking, the plasmoid chain is not a simple sequence of closed magnetic islands and current sheets. For example, while the left Y-point of a secondary layer located to the right of the global center separates it from a plasmoid to its left, the right Y-point separates it from a region of open flux followed by the plasmoid to its right (see Fig. 11).

We can now relate the open flux to the overall reconnection rate. The total reconnected flux is the sum of all open-flux parcels between the center of the global layer $(y=0)$ and its right end $(y=+L)$. Reconnection in any given secondary layer adds to the open flux on its right and subtracts from the open flux enveloping the plasmoid on its left. Upon summing up over all the $y>0$ layers, the contributions from all except for the very first one (closest to the global center) cancel. The total open flux is thus equal to the flux reconnected via that centralmost layer. Since in a statistical steady state each plasmoid and each open-flux parcel eventually get ejected, the net effective reconnection rate equals that of a single secondary layer: $E_{\text {eff }} \sim E^{(2)}$. Note that this argument is very general; it is purely kinematic and does not rely on any dynamical considerations (e.g., on our assumptions IIII). Applying the same reasoning to all levels $n$ in the self-similar plasmoid hierarchy, down to the critical layer at the bottom, we see that the effective reconnection rate is scale invariant (the same for all levels) and is equal to:

$$
c E_{\mathrm{eff}}=c E_{\mathrm{eff}}^{(n)} \sim c E_{c} \sim S_{c}^{-1 / 2} V_{A} B_{0} \sim 10^{-2} V_{A} B_{0} .
$$

This means that truly slow reconnection cannot occur, even in collisional plasmas. And indeed, we are not aware of any numerical or observational evidence for $\tilde{E}_{\text {eff }} \equiv$ $c E_{\text {eff }} / B_{0} V_{A}$ much below $10^{-2}$.

Plasmoid growth and flux distribution. We are interested in a more detailed statistical description of the hierarchical plasmoid chain, beyond just the effective reconnection rate. Namely, we want to find the plasmoid spectrum, i.e., the plasmoid flux and size distributions (c.f. [18]; see 16] for a numerical study of the statistics of current sheets). To do this, we analyze the inter- 
play between plasmoid growth, ejection, and coalescence. We first focus on "normal" plasmoids - those born not very close to the centers of their host layers (previousgeneration layers in which they are embedded). The importance of this distinction will become clear later.

Consider a normal plasmoid of class $n$ inside a host layer of length $2 L^{(n-1)}$ connecting two plasmoids of class $n-1$. The plasmoid lives until it is ejected from the host layer and merges (coalesces) with a larger plasmoid of a previous class. The ejection lifetime is $t_{\mathrm{ej}}^{(n)} \sim$ $L^{(n-1)} / V_{\text {out }}^{(n)}$, where $V_{\text {out }}^{(n)}$ is the typical plasmoid velocity within the host layer. Since, by corollary to assumption II, $V_{\text {out }}^{(n)} \sim V_{A}$ for all plasmoid classes [19], $t_{\mathrm{ej}}^{(n)} \sim \tau_{A}^{(n-1)}=L^{(n-1)} / V_{A}$. During this time, the plasmoid grows via reconnection in the two $n$ th-level layers on its sides. By assumption III (to be verified later), the plasmoid is ejected before saturating, so its flux grows as $d \Psi^{(n)} / d t=c E_{\mathrm{eff}}^{(n+1)} \sim c E_{\mathrm{eff}}$, and eventually reaches

$$
\Psi^{(n)} \sim c E_{\mathrm{eff}}^{(n+1)} t_{\mathrm{ej}}^{(n)} \sim c E_{\mathrm{eff}} L^{(n-1)} / V_{A} \sim \tilde{E}_{\mathrm{eff}} B_{0} L^{(n-1)} .
$$

We can now find the cumulative plasmoid-flux distribution function $N(\Psi)$ - the total number (in the global layer of length $2 L$ ) of plasmoids with fluxes larger than $\Psi$. On the one hand, the relationship between the typical flux $\Psi^{(n)}$ of normal plasmoids and their host-layer length $L^{(n-1)}$ is given by Eq. (2). On the other hand, $L^{(n-1)}$ is comparable to the typical separation between plasmoids larger than $\Psi^{(n)}$ (i.e., of all previous classes): $2 L^{(n-1)} \sim \Delta y^{(n-1)} \sim 2 L / N\left(\Psi^{(n)}\right)$. Dropping the class index $n$, we then get $\Psi N(\Psi) \sim c E_{\text {eff }} L / V_{A}=\tilde{E}_{\text {eff }} B_{0} L$, and hence the flux distribution density is

$$
f(\Psi)=-d N / d \Psi \sim \tilde{E}_{\mathrm{eff}} B_{0} L \Psi^{-2} .
$$

This is a testable quantitative result. Another way to derive it is to consider a plasmoid-dominated layer and randomly pick a plasmoid with some flux $\Psi_{0}$. Its expected age is $\tau_{\text {past }} \sim \Psi_{0} / c E_{\text {eff }}$ and its future life expectancy (the time before it is ejected into a larger plasmoid) is $\tau_{\text {future }} \sim$ $\Delta y\left(\Psi>\Psi_{0}\right) / V_{A} \sim L / V_{A} N\left(\Psi_{0}\right)$. Since the plasmoid was chosen randomly, we expect $\tau_{\text {future }} \sim \tau_{\text {past }}$ [20], which again yields $\Psi_{0} N\left(\Psi_{0}\right) \sim c E_{\text {eff }} L / V_{A}$.

Growth and distribution of plasmoid sizes. When a small amount of flux $\delta \Psi^{(n)}=B_{0} \delta x$ is reconnected and added to a growing plasmoid of class $n$, its area is increased by $\delta A^{(n)} \sim \Delta y^{(n)} \delta x=\Delta y^{(n)} \delta \Psi^{(n)} / B_{0}$, where $\Delta y^{(n)}$ is the separation between plasmoids of class $n$. On the normal-plasmoid life timescale $t_{\mathrm{ej}}^{(n)} \lesssim \tau_{A}^{(n-1)}, \Delta y^{(n)}$ is not changed strongly by the Hubble flow, $\Delta y^{(n)} \sim$ const. Then, the plasmoid area grows just as $A^{(n)} \sim$ $\Delta y^{(n)} \Psi^{(n)} / B_{0}$. As long as the plasmoid $x$-width $w_{x}^{(n)}$ remains smaller than its $y$-extent $w_{y}^{(n)}=\Delta y^{(n)}-2 L^{(n)}$, the latter stays roughly constant and comparable to $\Delta y^{(n)}$. Then, the growth of the plasmoid area translates directly into the growth of its $x$-width: $w_{x}^{(n)} \sim A^{(n)} / w_{y}^{(n)} \sim$ $\Psi^{(n)} / B_{0}$. Using Eq. (2), we find

$$
w_{x}^{(n)} \sim \frac{c E_{\mathrm{eff}}^{(n+1)} t_{\mathrm{ej}}^{(n)}}{B_{0}} \sim \frac{c E_{\mathrm{eff}}^{(n+1)} L^{(n-1)}}{B_{0} V_{\mathrm{out}}^{(n)}} \sim \tilde{E}_{\mathrm{eff}} L^{(n-1)} .
$$

Interestingly, this allows us to make a comparison with the Shibata-Tanuma [17] estimate for the reconnection rate $c E_{\mathrm{eff}}^{(S T)} \sim V_{\text {out }} B_{0} w_{\max } / L$, based on a $\mathrm{SP}$ mass conservation argument and the assumption that the effective outflow channel width is the plasmoid chain width $w_{\max }$. Applying their estimate to any $n$th level in the hierarchy and taking $w_{\max } \sim w_{x}^{(n)}$, given by Eq. (4), we get: $c E_{\mathrm{eff}}^{(n)} \sim V_{\mathrm{out}}^{(n)} B_{0} w_{x}^{(n)} / L^{(n-1)} \sim c E_{\mathrm{eff}}^{(n+1)}$, independent of $V_{\text {out }}$ or of $n$, which coincides with our result Eq. (1).

The size distribution of normal plasmoids now follows from Eq. (3) and the width-flux relation $w_{x}(\Psi) \sim \Psi / B_{0}$ :

$$
f\left(w_{x}\right)=-d N\left(w_{x}\right) / d w_{x} \sim \tilde{E}_{\text {eff }} L w_{x}^{-2} .
$$

Lack of saturation. These results allow us to verify our assumption III that plasmoids do not saturate. Saturation would occur if $w_{x}$ at any level became comparable with the typical separation $\Delta y(\Psi) \sim L / N(\Psi)$ between plasmoids of this size or larger. Using $w_{x} \sim \Psi / B_{0}$ and Eq. (3), we have $w_{x} / \Delta y(\Psi) \sim \Psi N(\Psi) / B_{0} L \sim \tilde{E}_{\text {eff }} \ll 1$, Thus, the nonlinear saturation never becomes an issue.

It is worth noting the crucial role of plasmoid coalescence (ejection into larger plasmoids) in mitigating nonlinear saturation. If we only had a simple chain of $N \sim L / L_{c} \sim S / S_{c}$ plasmoids moving along the global layer, then plasmoid growth would saturate at $w_{\text {sat }} \sim L_{c}$, corresponding to a plasmoid flux of $\Psi_{\text {sat }} \sim B_{0} L_{c}$. The time for this to happen, $t_{\text {sat }} \sim \Psi_{\text {sat }} / c E_{c} \sim\left(L_{c} / V_{A}\right) S_{c}^{1 / 2}$, would be shorter than the global ejection time $\tau_{A}=L / V_{A}$ if $S>S_{c}^{3 / 2} \sim 10^{6}$. Thus, without coalescence, plasmoids would quickly saturate, stifling reconnection for $S>S_{c}^{3 / 2}$. Coalescence prevents the accumulation of saturated plasmoids and allows larger plasmoids to grow in size by eating up smaller ones; it is thus essential for the fast plasmoid-dominated reconnection.

Monster plasmoids. The above picture is modified by the presence, in addition to the "normal" plasmoids, of relatively rare "anomalous" plasmoids born near the centers of their host layers at any level in the hierarchy. The most important of them are the lowest-generation anomalous plasmoids born near the center of the global layer of length $2 L$. Because the Hubble-flow ejection time for an anomalous plasmoid born at $y=y_{0} \ll L$ is $t_{\mathrm{ej}}=\int_{y_{0}}^{L} d y / v_{y} \simeq \tau_{A} \log \left(L / y_{0}\right)$, this plasmoid lives longer than normal plasmoids and hence grows larger. Its final flux is enhanced just by a logarithmic factor: $\Psi \sim c E_{\text {eff }} \tau_{A} \log \left(L / y_{0}\right)$; however, the enhancement of its area is much greater. Indeed, a given plasmoid grows in mass and area by sucking in all the plasma (including smaller plasmoids) within its domain of influence, 
which extends up to the mid-point between it and the next plasmoid of similar size. Thus, the area growth rate is proportional to the interplasmoid separation $\Delta y(t)$ : $d A / d t \sim\left[\Delta y(t) / B_{0}\right] d \Psi / d t \sim \Delta y(t) \tilde{E}_{\text {eff }} V_{A}$. But, on their long ejection timescale, the separation between two anomalous plasmoids grows exponentially, $\Delta y(t) \sim$ $\Delta y(0) \exp \left(t / \tau_{A}\right)$. Therefore, $A(t)$ also grows exponentially and ultimately reaches $A_{\max }=\int_{0}^{t_{\mathrm{ej}}} d t(d A / d t) \sim$ $\tilde{E}_{\text {eff }} L^{2} \Delta y(0) / y_{0}$, larger by a factor $L / y_{0} \gg 1$ than for normal plasmoids. Taking the typical smallest initial position $y_{0}$ to be $\sim \Delta y(0)$, we get $A_{\max } \sim \tilde{E}_{\text {eff }} L^{2}$. Next, it is easy to show that for $S>S_{c}^{5 / 4} \sim 10^{5}$, this growth is so rapid that the plasmoid $x$-width $w_{x}$ catches up with its initial $y$-extent $w_{y} \sim \Delta y(0)$ before $t_{\mathrm{ej}}$ is reached. Then, subsequently, $w_{x}$ and $w_{y}$ grow in unison and eventually reach $w_{\max } \sim A_{\max }^{1 / 2} \sim \tilde{E}_{\text {eff }}^{1 / 2} L \sim 0.1 L$. This prediction of occasional large, macroscopic "monster" plasmoids should have important implications for observations (e.g., large abrupt events in solar flares 21] and sawtooth crashes [2]) and simulations.

Transition to collisionless reconnection. Even if the global reconnection layer is in the resistive MHD regime, $\delta_{\mathrm{SP}}(L)>\min \left\{d_{i}, \rho_{s}\right\}$, where $d_{i}$ is the ion collisionless skin-depth and $\rho_{s}$ is the ion sound Larmor radius, this may not be so for the smaller layers in the plasmoid hierarchy. Namely, if $\delta_{c}<\min \left\{d_{i}, \rho_{s}\right\}$, then resistive MHD breaks down at some level in the hierarchy and the corresponding current sheets transition to fast collisionless reconnection with $c E_{\text {Hall }} \simeq 0.1 B_{0} V_{A} \sim 10 c E_{c}$. The hierarchy terminates at this point (c.f. 12, 16, 22]). Most of our results should still hold, with $E_{\text {eff }} \sim E_{\text {Hall }}$. Interestingly, the resulting range, $0.01 \lesssim \tilde{E}_{\text {eff }} \lesssim 0.1$, covers most of the rates inferred observationally or numerically.

One can evaluate the ratio $\delta_{c} / d_{i}$ as

$$
\delta_{c} / d_{i} \sim S_{c}^{1 / 2} \eta / V_{A} d_{i} \sim S_{c}^{1 / 2} \nu_{e} / \Omega_{e},
$$

where $\Omega_{e}$ and $\nu_{e}$ are the electron cyclotron and collision frequencies (c.f. 12]). Thus, the plasmoid hierarchy stays collisional all the way down to the critical layer only in very collisional plasmas. Using Spitzer resistivity, $\delta_{c} / d_{i} \sim\left(S_{c}^{1 / 2} / 12 \pi\right)\left(c / V_{A}\right)\left(m_{e} / m_{i}\right)^{1 / 2} \ln \Lambda N_{D}^{-1}$, where $N_{D} \equiv(4 \pi / 3) n_{e} \lambda_{D}^{3}$ is the number of electrons in a Debye sphere. Since $N_{D} \gg 1$ for a medium to be considered a normal plasma, we expect $\delta_{c} \ll d_{i}$ and so the smallest layers in the plasmoid chain are unavoidably collisionless. In the strong-guide-field case, $d_{i}$ is replaced by $\rho_{s}$ and the above expressions are only changed by factors of $\sim \beta^{1 / 2}$, which does not significantly affect our conclusions.

Conclusions. We have proposed a simple model of reconnection in plasmoid dominated current layers which yields a Lundquist-number independent effective reconnection rate $\tilde{E}_{\text {eff }} \sim S_{c}^{-1 / 2} \sim 0.01$ and a self-similar distribution of plasmoid sizes and fluxes (an inverse-square law, a testable prediction). In addition, we have argued that the plasmoid instability induces a multi-level plas- moid hierarchy that almost always reaches the kinetic scales, implying that pure resistive MHD reconnection occurs only in the most collisional plasmas. Our prediction of the occurrence of monster plasmoids, of width $w_{x} \sim 0.1 L$, offers a possible interpretation of observable events in solar flares and sawtooth crashes.

While a detailed nonlinear theory of plasmoid reconnection remains a challenge, as do fully resolved simulations of such a process, we hope that the simple model presented above might provide a useful conceptual framework for high-Lundquist-number reconnection in the way the SP model has done for moderate Lundquist numbers.

Acknowledgments. We thank R. Samtaney for important discussions. This work was supported by STFC (AAS), Fundação para a Ciência e Tecnologia, the European Communities under the contract between EURATOM and IST (NFL), and the Leverhulme Network for Plasma Turbulence. The views expressed herein do not necessarily reflect those of the European Commission.

[1] E. G. Zweibel and M. Yamada, Ann. Rev. Astron. Astrophys. 47, 291 (2009).

[2] H. K. Park et al., Phys. Rev. Lett. 96, 195003 (2006); T. Munsat et al., Nucl. Fusion 47, L31 (2007).

[3] M. Yamada, Phys. Plasmas 14, 058102 (2007); J. Egedal et al., Phys. Rev. Lett. 98, 015003 (2007).

[4] A. Retinò et al., Nat. Phys. 3, 236 (2007); J. Eastwood et al., J. Geophys. Res. 112, A06235 (2007).

[5] S. Chapman and P. C. Kendall, Proc. R. Soc. A 271, 435 (1963); S. I. Syrovatskii, Sov. Phys. JETP 33, 933 (1971); F. L. Waelbroeck, Phys. Rev. Lett. 70, 3259 (1993).

[6] N. F. Loureiro et al., Phys. Rev. Lett. 95, 235003 (2005).

[7] P. A. Sweet, in Electromagnetic Phenomena in Cosmical Physics, ed. B. Lehnert (1958), IAU Symposium, vol. 6, p. 123; E. N. Parker, J. Geophys. Res. 62, 509 (1957).

[8] N. F. Loureiro et al., Phys. Plasmas 14, 100703 (2007).

[9] R. Samtaney et al., Phys. Rev. Lett. 103, 105004 (2009).

[10] D. Biskamp, Phys. Fluids 29, 1520 (1986).

[11] G. Lapenta, Phys. Rev. Lett. 100, 235001 (2008).

[12] W. Daughton et al., Phys. Rev. Lett. 103, 065004 (2009).

[13] N. F. Loureiro et al., Mon. Not. R. Astron. Soc. 399, L146 (2009).

[14] A. Bhattacharjee et al., Phys. Plasmas 16, 112102 (2009).

[15] P. A. Cassak et al., Phys. Plasmas 16, 0702 (2009).

[16] Y. Huang and A. Bhattacharjee, Phys. Plasmas 17, 062104 (2010).

[17] K. Shibata and S. Tanuma, Earth Planets Space 53, 473 (2001).

[18] R. L. Fermo et al., Phys. Plasmas 17, 010702 (2010).

[19] D. A. Uzdensky et al., in preparation (2010).

[20] J. R. Gott III, Nature (London) 363, 315 (1993).

[21] J. Lin et al., Astrophys. J. 622, 1251 (2005); M. Karlický and B. Kliem (2010), arXiv:1007.2146.

[22] L. S. Shepherd and P. A. Cassak, Phys. Rev. Lett. 105, 015004 (2010).

[23] Note that this is much larger than the number of plasmoids in the linear regime, $N_{\text {lin }} \sim\left(S / S_{c}\right)^{3 / 8}$ [8] . 